\title{
DELAYED RESPONSES AND STABILITY IN TWO-SPECIES SYSTEMS
}

\author{
K. GOPALSAMY' \\ (Received I November 1982; revised 7 March 1983)
}

\begin{abstract}
It is shown that if intraspecific self-regulating negative feedback effects are strong enough such that a nontrivial steady state of a two species system is locally asymptotically stable, then time delays in the positive feedback as well as in other interspecific interactions cannot destabilise the system and hence delay induced instability leading to persistent oscillations is impossible whatever the magnitude of the time delays. A method is also proposed for an estimate of decay rate of perturbations.
\end{abstract}

\section{Preliminary remarks}

Almost all the equations of population ecology have been derived starting from the following simple format

$$
\frac{d N(t)}{d t}=\left\{\begin{array}{l}
\text { an individual's contribution } \\
\text { to population growth }
\end{array}\right\} N(t),
$$

where $N(t)$ denotes the size of a population of a single species (or biomass) with overlapping generations at time $t$, and $d N(t) / d t$ is the rate of change of the population size at time $t$. Subsequently one makes an assumption regarding the factor in the brackets in (1.1); in particular if one assumes that an individual's contribution to the population growth is denoted by a function say, $f(t, N)$, defined suitably for all $t>0, N \geqslant 0$ then one obtains from (1.1) the so called Kolmogorov formulation in the form

$$
\frac{d N(t)}{d t}=f(t, N(t)) N(t)
$$

\footnotetext{
'School of Mathematical Sciences, Flinders University, Bedford Park, S.A. 5042.

(C) Copyright Australian Mathematical Society 1984, Serial-fee code 0334-2700/84
} 
Various choices of $f$ together with some ecologically plausible assumptions such as the temporal constancy of the environment and density dependent effects in $f$ lead to various equations. For instance if $f(t, N) \equiv r$ (a positive constant) one obtains the Malthusian formulation in the form

$$
\frac{d N(t)}{d t}=r N(t)
$$

and if one assumes $f(t, N) \equiv r-(r / K) N$ fớ sôme positive cunstantis $r$ and $K$ one gets the familiar logistic equation of population biology

$$
\frac{d N(t)}{d t}=r N(t)\left\{1-\frac{N(t)}{K}\right\} \text {. }
$$

In order to derive models with time delayed effects, such effects are introduced in the average growth rate $f(t, N)$ of (1.2); for instance if $f(t, N) \equiv r-$ $(r / K) N(t-\tau)$ for some constant $\tau>0$ we obtain Hutchinson's [19] delay logistic equation

$$
\frac{d N(t)}{d t}=r N(t)\left\{1-\frac{N(t-\tau)}{K}\right\}
$$

If for instance

$$
f(t, N)=r-(r / K) N(t)-\int_{-\infty}^{t} k(t-s) F(N(s)) d s
$$

where $k$ is a piecewise continuous nonnegative valued function such that $\int_{0}^{\infty} k(s) d s=1$ with some appropriate function $F$, we will get Volterra's model of a population which pollutes its environment and pollution has an accumulative and toxic effect; such an equation is of the form

$$
\frac{d N(t)}{d t}=N(t)\left\{r-(r / K) N(t)-\int_{-\infty}^{t} k(t-s) F(N(s)) d s\right\}
$$

which has been studied in different formats by several authors (Cushing [12], Cohen et al. [10], Gopalsamy [16]).

One of the significant advantages of deriving various models from the prototype (1.2) is that if the intitial conditions (initial population sizes) are nonnegative then the nonnegativity of the population size $N(t)$ for $t>0$ follows from the fact that all the solutions of (1.2) satisfy

$$
N(t)=N(0) \exp \left[\int_{0}^{t} f(s, N(s)) d s\right]
$$

which implies that $N(t) \geqslant 0$ if $N(0) \geqslant 0$.

While the time delayed logistic equation (1.3) explains some of the observed oscillations of certain populations, its derivation has not been entirely satisfactory. For instance it has been pointed out by Ricklefs ([27], page 488) that " time lags might be expected to occur primarily in stabilising processes involving 
reproduction rather than death because death is an immediate response to environmental change". Recently Cushing [13] has examined the effects of response and maturation delays in a number of single and multispecies population models; as it has been noted by Cushing [13], the models involving delayed reproduction are not necessarily derivable from the Kolmogorov type systems of the form (1.2) by suitably defining the average growth rate $(1 / N)(d N / d t)=$ $f(t, N(t))$. While Cushing [13] has been mostly concerned with the possibility of delay induced oscillations we will consider a class of delays in reproduction and interspecific interaction which maintain the stability of the systems.

Our starting point to derive models with delayed reproduction is the following balance equation which assumes that there is no immigration or emigration.

$$
\frac{d N(t)}{d t}=\text { birth rate }- \text { death rate. }
$$

Now for instance if we consider a population of adult flies then the birth rate at time $t$ depends on the adult population at time $t-\tau$ where $\tau$ is the time required for the larvae to become adult flies. If the birth and death rates are governed by density dependent factors then we have from (1.5) the following time delayed model

$$
\frac{d N(t)}{d t}=b(N(t-\tau))-m(N(t))
$$

where the functions $b$ and $m$ denote the density dependent birth and death rates respectively. If the time delay is continuously distributed then one can consider in the place of (1.6) an equation of the form

$$
\frac{d N(t)}{d t}=b\left(\int_{-\infty}^{t} k(t-s) N(s) d s\right)-m(N(t))
$$

or equivalently

$$
\frac{d N(t)}{d t}=b\left(\int_{0}^{\infty} k(s) N(t-s) d s\right)-m(N(t))
$$

where the delay kernel $k$ denotes the weight of the delayed effects.

It is not always obvious that suitable nonnegative initial conditions for (1.6) and (1.7) will imply the nonnegativity of the solutions of (1.6) and (1.7). The purpose of this article is to show that in two species systems of competitive and prey-predator communities, delayed reproduction and delayed interactions do not destabilise the systems if the self-regulating negative feedback effects are sufficiently strong compared to interspecific interactive effects and if the self regulating effects are realised with no time delays. When we say that time delays do not destabilise, we will mean that delays do not render an asymptotically (locally) stable steady state unstable in such a manner that the loss of stability either leads 
to a delay-induced bifurcation to persistent and undamped oscillations (as in the case of Hopf-bifurcation) or the relevant linear variational system has unbounded solutions; that is a mathematical instability is not induced by delays in the sense, that the roots of the characteristic equation are pushed to the imaginary axis or to the right of it on the complex plane. However delays can make an otherwise stable system less stable which means that the rate of convergence to zero of solutions of the variational system can decrease with an increase of delay and the variational system can even oscillate (perhaps violently) before converging to the trivial state. Such a reduction in stability can happen even when all the roots of the characteristic equation have negative real parts. For our purposes such a reduced stability is still stability. In order to see the effects of delays on such less stable systems we will propose below a method of estimating the rate of decay of local perturbations based on the variational system and indirectly relying on the characteristic equation. For a special class of two species models with a single delay we have obtained (Gopalsamy [16]) estimates of decay rates (or return times) using the characteristic equation; such a procedure is almost impossible in the case of many species systems with several delays. The method we propose in Section 6 below for the estimate of decay rates can be adapted to $n$-species systems with $n^{2}$ discrete delays.

\section{Two species competition}

Let $x_{1}(t)$ and $x_{2}(t)$ denote the population sizes (or biomasses) of two species competing for a common pool of resources in a constant environment. Let $b_{i}$ and $m_{i}(i=1,2)$ denote the respective density dependent birth (potential) and death rates so that in the absence of any time delays the population sizes are governed by

$$
\left.\begin{array}{l}
\frac{d x_{1}(t)}{d t}=b_{1}\left(x_{1}(t)\right)-m_{1}\left(x_{1}(t), x_{2}(t)\right), \\
\frac{d x_{2}(t)}{d t}=b_{2}\left(x_{2}(t)\right)-m_{2}\left(x_{1}(t), x_{2}(t)\right) .
\end{array}\right\}
$$

In order to make the system (2.1) represent a competition system we will make the following assumptions on the birth and death rates;

(i) $b_{1}, m_{t}(i=1,2)$ are continuous with continuous partial derivatives for all $x_{t} \geqslant 0(i=1,2) ;$ also we assume (see for instance Rescigno and Richardson [26])

$$
\frac{\partial b_{i}}{\partial x_{i}}>0, \quad \frac{\partial m_{i}}{\partial x_{j}}>0 \quad \text { for } x_{i}>0 \quad(i, j=1,2),
$$


(ii)

$$
\left.\begin{array}{l}
b_{1}(0)=0, \quad m_{1}\left(0, x_{2}\right) \equiv 0, \\
b_{2}(0)=0, \quad m_{2}\left(x_{1}, 0\right) \equiv 0,
\end{array}\right\}
$$

(iii) for some $x_{1}^{*}>0, x_{2}^{*}>0$ we have

$$
\left.\begin{array}{l}
b_{1}\left(x_{1}^{*}\right)-m_{1}\left(x_{1}^{*}, 0\right)=0, \\
b_{2}\left(x_{2}^{*}\right)-m_{1}\left(0, x_{2}^{*}\right)=0
\end{array}\right\}
$$

(iv) there exist positive constants $\delta_{1}, \delta_{2}$ such that

$$
\left.\begin{array}{l}
b_{1}\left(\delta_{1}\right)-m_{1}\left(\delta_{1}, x_{2}\right)<0, \\
b_{2}\left(\delta_{2}\right)-m_{2}\left(x_{1}, \delta_{2}\right)<0,
\end{array}\right\} \text { for } x_{1} \geqslant 0, \quad x_{2} \geqslant 0,
$$

(v) there exists a point $(\alpha, \beta), \alpha>0, \beta>0$ such that

$$
b_{1}(\alpha)-m_{1}(\alpha, \beta)=0=b_{2}(\beta)-m_{2}(\alpha, \beta) .
$$

The conditions on $b_{i}$ in (2.2) mean that the potential (not average) birth rates are density dependent and any negative density dependent feedbacks to the birth rates are included in the death rates; the conditions on $m_{t}$ indicate intraspecific and interspecific competition. (2.3) implies that $(0,0)$ is a (trivial) steady state of the system (2.1) as it is in the case of all ecological models; (2.4) means that in the absence of either of the two species the other one has a nontrivial steady state; (2.5) implies that each of the two populations cannot grow unboundedly since when $x_{i}(t)>\delta_{i}, d x_{t} / d t<0$. (2.6) means that there exists a nontrivial steady state $(\alpha, \beta)$ in the interior of the positive quadrant of the $\left(x_{1}, x_{2}\right)$-plane. These assumptions are standard and are minimal in the opinion of the author.

An immediate example of (2.1)-(2.6) is the familiar Volterra-Lotka competition system described by

$$
\left.\begin{array}{l}
\frac{d x_{1}(t)}{d t}=r_{1} x_{1}(t)-a_{11} x_{1}^{2}(t)-a_{12} x_{1}(t) x_{2}(t), \\
\frac{d x_{2}(t)}{d t}=r_{2} x_{2}(t)-a_{21} x_{1}(t) x_{2}(t)-a_{22} x_{2}^{2}(t),
\end{array}\right\}
$$

where $r_{t}, a_{i j},(i, j=1,2)$ are positive constants satisfying the condition

$$
\frac{a_{11}}{a_{21}}>\frac{r_{1}}{r_{2}}>\frac{a_{12}}{a_{22}} .
$$

One of the first questions to be resolved for (2.1) is the following: if $x_{1}(0)>0$, $x_{2}(0)>0$ does it follow that the corresponding solutions of (2.1) satisfy the condition $x_{1}(t) \geqslant 0, x_{2}(t) \geqslant 0$ for those $t$ for which a solution of (2.1) exists? In the $\left(x_{1}, x_{2}\right)$ phase plane of $(2.1)$ we have $(0,0),\left(x_{1}^{*}, 0\right),\left(0, x_{2}^{*}\right),(\alpha, \beta)$ as the steady states of (2.1) and also it will follow from (2.3) that the coordinate axes 
$x_{1}=0$ and $x_{2}=0$ are invariant sets of (2.1); such invariance of the coordinate axes will imply that if $x_{1}(0)>0, x_{2}(0)>0$ then the corresponding population trajectory $\left(x_{1}(t), x_{2}(t)\right)$ of $(2.1)$ cannot reach the outside of the nonnegative quadrant $x_{1} \geqslant 0, x_{2} \geqslant 0$.

Our plan is to derive first a set of sufficient conditions for the coexistence of the two competing species and then in the next section show that under the same conditions the two species can coexist if there are time delays in the reproduction and interspecific interactions. We recall that the two competing species described by (2.1) are said to coexist if and only if the nontrivial steady state $(\alpha, \beta)$ is (at least locally) asymptotically stable. To examine such a stability of $(\alpha, \beta)$ we derive the corresponding linear variational system for the perturbations $X_{1}, X_{2}$ where

$$
x_{1}(t) \equiv \alpha+X_{1}(t), \quad x_{2}(t)=\beta+X_{2}(t) ;
$$

such a variational system is found to be

$$
\left.\begin{array}{l}
\frac{d X_{1}(t)}{d t}=\beta_{11} X_{1}(t)+\beta_{12} X_{2}(t), \\
\frac{d X_{2}(t)}{d t}=\beta_{21} X_{1}(t)+\beta_{22} X_{2}(t),
\end{array}\right\}
$$

where

$$
\beta_{l j}=\left(\frac{\partial b_{i}}{\partial x_{j}}-\frac{\partial m_{i}}{\partial x_{j}}\right) \text { evaluated at }(\alpha, \beta) \quad(i, j=1,2) .
$$

The steady state $(\alpha, \beta)$ of $(2.1)$ is locally asymptotically stable if and only if the trivial solution $(0,0)$ of $(2.8)$ is asymptotically stable. We can derive the following result from elementary considerations.

In the system (2.1)-(2.6) assume the following:

$$
\left.\begin{array}{l}
\frac{\partial m_{1}}{\partial x_{1}}>\frac{\partial b_{1}}{\partial x_{1}}+\frac{\partial m_{2}}{\partial x_{1}}, \\
\frac{\partial m_{2}}{\partial x_{2}}>\frac{\partial b_{2}}{\partial x_{2}}+\frac{\partial m_{1}}{\partial x_{2}},
\end{array}\right\} \quad \text { at }(\alpha, \beta) .
$$

Then the steady state $(\alpha, \beta)$ is (locally) asymptotically stable for (2.1)-(2.6).

The proof follows easily if we note that the characteristic equation associated with the linear system (2.8) is given by

$$
\operatorname{Det}\left[\begin{array}{rr}
\lambda-\beta_{11} & -\beta_{12} \\
-\beta_{21} & \lambda-\beta_{22}
\end{array}\right]=0
$$

or equivalently,

$$
\lambda^{2}-\lambda\left(\beta_{11}+\beta_{22}\right)+\beta_{11} \beta_{22}-\beta_{12} \beta_{21}=0 .
$$


It follows from (2.2) and (2.10) that,

$$
\beta_{11}<0, \beta_{22}<0, \beta_{11} \beta_{22}-\beta_{12} \beta_{21}>0
$$

and hence the roots of (2.11) have negative real parts implying the asymptotic stability of the trivial solution of (2.8).

If we apply the condition (2.10) to the Volterra-Lotka model (2.7) we note that when a nontrivial steady state say $(\alpha, \beta)$ exists for $(2.7),(2.10)$ leads to

$$
a_{11} \alpha>a_{21} \beta \text { and } a_{22} \beta>a_{12} \alpha,
$$

which together imply $a_{11} a_{22}>a_{12} a_{21}$. If $(\alpha, \beta)$ exists it is known that $a_{11} a_{22}-$ $a_{12} a_{21}>0$ is a sufficient condition for the (local) asymptotic stability of $(\alpha, \beta)$.

The condition (2.10) which guarantees the coexistence of competing populations in (2.1)-(2.6) simply means that the intraspecific self-regulating feedback effects $\partial m_{i} / \partial x_{t}(i=1,2)$ are higher than its own positive feedback $\partial b_{t} / \partial x_{t}$ $(i=1,2)$ together with its negative (competitive) effects on its competitor $\partial m_{l} / \partial x_{\text {, }}$ $(i, j=1,2 ; i \neq j)$.

\section{Coexistence under delayed reproduction and interactions}

We will now establish that two competing species with a sufficiently strong intraspecific regulation can coexist in the presence of delayed reproductions and delayed interspecific interactions. It is well known that if there are delays in the self-regulating intraspecific effects, stationary coexistence may not always be possible (Gopalsamy [16], Gopalsamy and Aggarwala [15], Shibata and Saito [28]). Delayed reproduction is more general and widespread in most biological populations. To build a competition model with such delays we proceed as follows.

Let $\tau_{i j}(i, j=1,2)$ be a set of nonnegative constants with $\tau=\max \tau_{i j}, i, j=$ 1,2 , and suppose that the two competing species display delayed reproduction and delayed interspecific interactions with no delays in the self-regulating negative feedback effects. Such a competition system in a constant environment can be modelled by the autonomous delay differential system

$$
\left.\begin{array}{l}
\frac{d x_{1}(t)}{d t}=b_{1}\left(x_{1}\left(t-\tau_{11}\right)\right)-m_{1}\left(x_{1}(t), x_{2}\left(t-\tau_{12}\right)\right), \\
\frac{d x_{2}(t)}{d t}=b_{2}\left(x_{2}\left(t-\tau_{22}\right)\right)-m\left(x_{1}\left(t-\tau_{21}\right), x_{2}(t)\right),
\end{array}\right\}
$$

where both the birth rates and the mortality rates $b_{t}$ and $m_{t}(i=1,2)$ satisfy the same conditions (2.2)-(2.6) as in the previous section. Along with (3.1) we will 
suppose that the initial population sizes are provided in the form

$$
x_{i}(s)=\phi_{i}(s) \geqslant 0, \quad s \in[-\tau, 0], \quad i=1,2 .
$$

(Note that the specification of $\phi_{i}$ need not be on the entire interval $[-\tau, 0]$; but such a hypothesis is convenient.) Since (3.1) is not of the standard Kolmogorovtype, we have to establish that the solutions of (3.1) will remain nonnegative for nonnegative initial conditions.

To prove the nonnegativity of solutions of (3.1)-(3.2), let $\left\{\psi_{1}^{(n)}(s)\right\}, s \in[-\tau, 0]$, $i=1,2 ;(n=1,2, \ldots)$ be a sequence of strictly positive continuous functions such that

$$
\lim _{n \rightarrow \infty} \psi_{i}^{(n)}(s)=\psi_{i}(s), \quad s \in[-\tau, 0], \quad i=1,2 .
$$

Let $\left\{x_{1}^{(n)}(t), x_{2}^{(n)}(t)\right\}$ be the solution of (3.1) corresponding to the initial condition

$$
\left\{x_{1}^{(n)}(s), x_{2}^{(n)}(s)\right\}=\left\{\psi_{1}^{(n)}(s), \psi_{2}^{(n)}(s)\right\}, \quad s \in[-\tau, 0] .
$$

Consider the solution $\left\{x_{1}^{(n)}(t), x_{2}^{(n)}(t)\right\}$ for $t \in[0, \tau]$ (assuming it exists on the whole of $[0, \tau])$. Suppose $x_{1}^{(n)}(t)$ does not remain nonnegative for all $\left[0, \tau^{*}\right]$; then there exists a $t^{*}=\left[0, \tau^{*}\right]\left(\tau^{*}=\right.$ the positive minimum of $\left.\tau_{i j}(i, j=1,2)\right)$ for which

$$
x_{1}^{(n)}\left(t^{*}\right)=0 \quad \text { and } \quad x_{1}^{(n)}(t)\left(t^{*}-\tau_{11}\right)>0 .
$$

It will follow from (3.1) and the positivity of the initial condition that

$$
\frac{d x_{1}^{(n)}\left(t^{*}\right)}{d t}=b_{2}\left(x_{1}^{(n)}\left(t^{*}-\tau_{11}\right)\right)>0
$$

where we have used the properties of $b_{i}$ and $m_{i}$ from (2.2)-(2.6); it is found that (3.5) contradicts the definition of $t^{*}$. Thus $x_{1}^{(n)}(t)>0$ on $\left[0, \tau^{*}\right]$; similarly $x_{2}^{(n)}(t)>0$ on $\left[0, \tau^{*}\right]$. We can repeat this procedure for intervals of the form $\left[\tau^{*}, 2 \tau^{*}\right],\left[2 \tau^{*}, 3 \tau^{*}\right]$ etc. Thus it will follow that so long as $\left(x_{1}^{(n)}(t), x_{2}^{(n)}(t)\right)$ exists, we have

$$
x_{1}^{(n)}(t) \geqslant 0, \quad x_{2}^{(n)}(t) \geqslant 0 .
$$

If we now consider the limits as $n \rightarrow \infty$, we get

$$
\lim _{n \rightarrow \infty}\left\{x_{1}^{(n)}(t), x_{2}^{(n)}(t)\right\}=\left\{x_{1}(t), x_{2}(t)\right\}
$$

with $x_{1}(t) \geqslant 0, x_{2}(t) \geqslant 0$ where $\left(x_{1}(t), x_{2}(t)\right)$ is the solution of (3.1)-(3.2) and this is a consequence of the continuous dependence of solutions of (3.1) on initial conditions (Hale [17], page 41).

A second question for (3.1) with (2.2)-(2.6) is whether solutions of (3.1) exist for all $t>0$ ? Suppose now that solutions of (3.1) do not exist for all $t \geqslant 0$; then 
there exists a $t_{1}>0$ such that at least for one of either of $x_{1}$ or $x_{2}$ we have

$$
\lim _{t \rightarrow t_{1^{-}}} x_{i}(t)=\infty \text {. }
$$

To be specific let us suppose that $\lim _{t \rightarrow t_{1}-} x_{1}(t)=\infty$; let $t_{2}$ be the first instant of time for which $x_{1}\left(t_{2}\right)=\delta_{1}, t_{2}<t_{1}$. It will follow from (3.1) that

$$
\begin{aligned}
\frac{d x_{1}\left(t_{2}\right)}{d t} & =b_{1}\left(x_{1}\left(t_{2}-\tau_{11}\right)\right)-m_{1}\left(x_{1}\left(t_{2}\right), x_{2}\left(t_{2}-\tau_{12}\right)\right) \\
& <b_{1}\left(x_{1}\left(t_{2}\right)\right)-m_{1}\left(x_{1}\left(t_{2}\right), x_{2}\left(t_{2}-\tau_{12}\right)\right) \\
& <b_{1}\left(\delta_{1}\right)-m_{1}\left(\delta_{1}, x\left(t_{2}-\tau_{12}\right)\right)<0
\end{aligned}
$$

which contradicts the definition of $t_{2}$. Thus solutions of $(3.1),(2.2)-(2.6)$ exist for all $t \geqslant 0$.

We are now ready to consider the effects of time delays in reproduction and interspecific interactions on the coexistence of the two competing species. The steady state $(\alpha, \beta)$ of $(2.1)$ is again a steady state of (3.1). To examine the (local) asymptotic stability of $(\alpha, \beta)$ in (3.1) we let

$$
x_{1}(t)=\alpha+Y_{1}(t), \quad x_{2}(t)=\beta+Y_{2}(t)
$$

in (3.1) and derive that the linear variational system governing the perturbations $Y_{1}, Y_{2}$ is given by

$$
\left.\begin{array}{l}
\frac{d Y_{1}(t)}{d t}=-m_{11} Y_{1}(t)+b_{11} Y_{1}\left(t-\tau_{11}\right)-m_{12} Y_{2}\left(t-\tau_{12}\right), \\
\frac{d Y_{2}(t)}{d t}=-m_{22} Y_{2}(t)-m_{21} Y_{1}\left(t-\tau_{21}\right)+b_{22} Y_{2}\left(t-\tau_{22}\right),
\end{array}\right\}
$$

where

$$
m_{i j}=\frac{\partial m_{i}}{\partial x_{j}}, \quad b_{i i}=\frac{\partial b_{i}}{\partial x_{i}} \quad \text { at }(\alpha, \beta) .
$$

It is well known (Bellman and Cooke [3], page 336) that the steady state $(\alpha, \beta)$ is (locally) asymptotically stable for (3.1) if the trivial solution of the variational system (3.9) is asymptotically stable. We formulate our first result in the following form whose proof is given in the appendix.

THEOREM 3.1. Assume that in the two species competition model system (3.1) with delayed density dependent birth and death rates $b_{i}$ and $m_{i}(i=1,2)$ satisfy the conditions (2.2)-(2.6). Suppose that the conditions (2.10) hold. Then the nontrivial steady state $(\alpha, \beta)$ is (locally) asymptotically stable for (3.1) whatever be the delays $\tau_{i j} \geqslant 0(i=1,2)$. 


\section{A prey-predator system}

Let $y_{1}(t)$ and $y_{2}(t)$ respectively denote the population sizes (or biomasses) of a prey species and a predator species which feeds exclusively on the single prey species in a temporally constant environment. In the format of (1.5) the following system will describe the rates of change of the two population sizes:

$$
\left.\begin{array}{l}
\frac{d y_{1}(t)}{d t}=f_{1}\left(y_{1}(t)\right)-g_{1}\left(y_{1}(t), y_{2}(t)\right) \\
\frac{d y_{2}(t)}{d t}=f_{2}\left(y_{1}(t), y_{2}(t)\right)-g_{2}\left(y_{2}(t)\right)
\end{array}\right\}
$$

where $f_{t}$ and $g_{l}(i=1,2)$ respectively denote the birth and death rates of the two species; in order to make (4.1) denote the dynamics of a prey-predator species we will assume that $f_{i}$ and $g_{\imath}$ satisfy the following conditions (see for instance Rescigno and Richardson [26]);

(i) $f_{t}$ and $g_{1}$ are continuous with continuous partial derivatives for all $y_{i} \geqslant 0$ $(i=1,2)$; also we require

$$
\left.\begin{array}{lll}
\frac{\partial f_{1}}{\partial y_{1}}>0 ; & \frac{\partial g_{1}}{\partial y_{1}} \geqslant 0 ; & \frac{\partial g_{1}}{\partial y_{2}} \geqslant 0, \\
\frac{\partial f_{2}}{\partial y_{1}} \geqslant 0 ; & \frac{\partial f_{2}}{\partial y_{2}} \geqslant 0 ; & \frac{\partial g_{2}}{\partial y_{2}}>0,
\end{array}\right\} \quad \text { where } y_{1} \geqslant 0, y_{2} \geqslant 0,
$$

(ii)

$$
\left.\begin{array}{rr}
f_{1}(0)=0, & g_{1}\left(0, y_{2}\right) \equiv 0, \\
f_{2}\left(0, y_{2}\right)=0=f_{2}\left(y_{1}, 0\right) ; & g_{2}(0)=0,
\end{array}\right\}
$$

(iii) for some constant $y_{1}^{*}>0$ we have

$$
f_{1}\left(y_{1}^{*}\right)-g_{1}\left(y_{1}^{*} 0\right)=0
$$

(iv) there exists a point $\left(\alpha^{*}, \beta^{*}\right), \alpha^{*}>0, \beta^{*}>0$ such that

$$
\left.\begin{array}{l}
f_{1}\left(\alpha^{*}\right)-g_{1}\left(\alpha^{*}, \beta^{*}\right)=0, \\
f_{2}\left(\alpha^{*}, \beta^{*}\right)-g_{2}\left(\beta^{*}\right)=0,
\end{array}\right\}
$$

(v) there exist positive constants $\eta_{1}, \eta_{2}$ such that

$$
\left.\begin{array}{l}
f_{1}\left(\eta_{1}\right)-g_{1}\left(\eta_{1}, y_{2}\right)<0, \quad y_{2} \geqslant 0, \\
f_{2}\left(\eta_{1}, \eta_{2}\right)-g_{2}\left(\eta_{2}\right)<0 .
\end{array}\right\}
$$

The conditions (4.2) mean that the system (4.1) is of the prey-predator type; (4.3) will imply that $(0,0)$ is a trivial steady state of $(4.1) ;(4.4)$ means that in the absence of the predator, the prey species has a nontrivial (positive) steady state; 
(4.5) implies that (4.1) has a nontrivial steady state in the interior of the positive quadrant of the state species of $(4.1) ;(4.6)$ can be shown (see below) to imply that no species can grow unboundedly.

An example of (4.1)-(4.6) is the familiar Volterra-Lotka prey-predator system

$$
\begin{aligned}
& \frac{d y_{1}(t)}{d t}=r_{1} y_{1}(t)-\left[a_{11} y_{1}^{2}(t)+a_{12} y_{1}(t) y_{2}(t)\right], \\
& \frac{d y_{2}(t)}{d t}=a_{21} y_{1}(t) y_{2}(t)-\left[r_{2} y_{2}(t)+a_{22} y_{2}^{2}(t)\right],
\end{aligned}
$$

where $r_{i}, a_{i j}(i, j=1,2)$ are positive constants such that

$$
a_{21} r_{1}-r_{2} a_{11}>0 \text {. }
$$

As in the previous case of competition we have to verify that when $y_{1}(0)>0$, $y_{2}(0)>0$ the corresponding solutions of (4.1) will be nonnegative for $t \geqslant 0$ for which the solutions exist. Since the co-ordinate axes $y_{1}=0, y_{2}=0$ are invariant sets for (4.1) by virtue of (4.3), it will follow that the solutions of (4.1) starting from the interior of the positive quadrant cannot reach the outside of that quadrant. This is a consequence of the invariance of the co-ordinate axes for (4.1).

To prove that solutions of (4.1)-(4.6) exist for all $t \geqslant 0$ let us suppose that $y_{1}(0)$ and $y_{2}(0)$ satisfy $0<y_{1}(0)<\eta_{1}, 0<y_{2}(0)<\eta_{2}$ (on the other hand if either one of $y_{1}(0) \geqslant \eta_{1}$ and $y_{2}(0) \geqslant \eta_{2}$ or both hold, it will follow that the corresponding $y_{1}(t)$ will decrease by virtue of (4.6) at least for small $t \geqslant 0)$. Suppose $y_{1}(t)$ does not exist for all $t \geqslant 0$; then for some $t_{1}<\infty, \lim _{t \rightarrow t_{4}-} y_{1}(t)=\infty$ and let $t_{2}>0$ be the first instant for which $y_{1}\left(t_{2}\right)=\eta_{1}$; then we have from (4.1) and (4.6) that

$$
\left.\frac{d y_{1}\left(t_{2}\right)}{d t}=f_{1}\left(\eta_{1}\right)-g_{1}\left(\eta_{1}, y_{2}\right)<0 \quad \text { (assuming } y_{1}(0)<\eta_{1}\right)
$$

which contradicts the definition of $t_{2}$ showing that $y_{1}(t)$ exists for all $t \geqslant 0$; if $y_{2}(t)$ is not defined for all $t \geqslant 0$, then for some $t_{3}<\infty$ we will have $\lim _{t \rightarrow t_{3}-} y_{2}(t)$ $=\infty$; now let $t_{4}$ be the first instant for which $y_{2}\left(t_{4}\right)=\eta_{2}$; then from the properties of $f_{2}$ we have

$$
\begin{aligned}
\frac{d y_{2}\left(t_{4}\right)}{d t} & =f_{2}\left(y_{1}\left(t_{4}\right), \eta_{2}\right)-g_{2}\left(\eta_{2}\right) \leqslant f_{2}\left(\eta_{1}, \eta_{2}\right)-g_{2}\left(\eta_{2}\right) \\
& \left.<0 \quad \text { (by (4.6) assuming } 0<y_{1}(0)<\eta_{1}, 0<y_{2}(0)<\eta_{2}\right)
\end{aligned}
$$

which again contradicts the definition of $t_{4}$. Thus $y_{2}(t)$ is defined for all $t \geqslant 0$.

We will now derive a set of sufficient conditions for the (local) asymptotic stability of the steady state $\left(\alpha^{*}, \beta^{*}\right)$ of (4.1). In the next section we will show that under the same conditions and in the presence of various delays, the steady state $\left(\alpha^{*}, \beta^{*}\right)$ remains (locally) asymptotically stable. 
To examine the asymptotic stability of the nontrivial steady state $\left(\alpha^{*}, \beta^{*}\right)$ in (4.1) we let

$$
y_{1}(t)=\alpha^{*}+U_{1}(t), \quad y_{2}(t)=\beta^{*}+U_{2}(t)
$$

in (4.1) and derive the linear variational system in the perturbations $U_{1}, U_{2}$ as follows:

$$
\left.\begin{array}{l}
\frac{d U_{1}(t)}{d t}=\frac{\partial f_{1}}{\partial y_{1}} U_{1}(t)-\frac{\partial g_{1}}{\partial y_{1}} U_{1}(t)-\frac{\partial g_{1}}{\partial y_{2}} \bar{U}_{2}(t), \\
\frac{d U_{2}(t)}{d t}=\frac{\partial f_{2}}{\partial y_{1}} U_{1}(t)+\frac{\partial f_{2}}{\partial y_{2}} U_{2}(t)-\frac{\partial g_{2}}{\partial y_{2}} U_{2}(t),
\end{array}\right\}
$$

where all the partial derivatives in (4.7) are evaluated at $\left(\alpha^{*}, \beta^{*}\right)$. The steady state $\left(\alpha^{*}, \beta^{*}\right)$ of (4.1) is (locally) asymptotically stable if and only if the trivial solution of (4.7) is asymptotically stable which will be the case if and only if the roots of the characteristic equation

$$
\begin{aligned}
\operatorname{Det}\left[\begin{array}{cc}
\lambda-\left(f_{11}-g_{11}\right) & g_{12} \\
-f_{21} & \lambda-\left(f_{22}-g_{22}\right)
\end{array}\right] & =0 \\
& \text { where }\left\{\begin{array}{c}
f_{i j}=\frac{\partial f_{i}}{\partial y_{j}}, \\
g_{i j}=\frac{\partial g_{1}}{\partial y_{j}}, \\
(i, j=1,2),
\end{array}\right\} \text { at }\left(\alpha^{*}, \beta^{*}\right)
\end{aligned}
$$

have negative real parts. If $\lambda_{1}, \lambda_{2}$ are the roots of (4.8) then

$$
\left.\begin{array}{r}
\lambda_{1}+\lambda_{2}=\left(f_{11}-g_{11}\right)+\left(f_{22}-g_{22}\right), \\
\lambda_{1} \lambda_{2}=\left(f_{11}-g_{11}\right)\left(f_{22}-g_{22}\right)+f_{21} g_{12} \cdot
\end{array}\right\}
$$

The proof of the following result is an immediate consequence of (4.7)-(4.9).

THEOREM 4.1. In the prey-predator system (4.1)-(4.6) assume that the partial derivatives satisfy the following additional conditions:

$$
\left.\begin{array}{l}
\frac{\partial g_{1}}{\partial y_{1}}>\frac{\partial f_{1}}{\partial y_{1}}+\frac{\partial f_{2}}{\partial y_{1}}, \\
\frac{\partial g_{2}}{\partial y_{2}}>\frac{\partial f_{2}}{\partial y_{2}}+\frac{\partial g_{1}}{\partial y_{2}},
\end{array}\right\} \quad \text { evaluated at }\left(\alpha^{*}, \beta^{*}\right)
$$

then the steady state $\left(\alpha^{*}, \beta^{*}\right)$ is (locally) asymptotically stable for the prey-predator system. 


\section{Delays in reproduction, predation and gestation}

A number of authors have proposed and analysed several models of prey-predator systems with time delays. Most of the models have been derived from the so called models of the Kolmogorov-type of (1.2) with time delays incorporated in the average growth rates of the prey and predator species. Volterra [32] proposed the following model for a prey-predator system

$$
\left.\begin{array}{l}
\frac{d N_{1}(t)}{d t}=N_{1}(t)\left[r_{1}-\delta_{1} N_{1}(t)-\int_{-\infty}^{t} F_{1}(t-s) N_{2}(s) d s\right], \\
\frac{d N_{2}(t)}{d t}=N_{2}(t)\left[-r_{2}+\delta_{2} N_{1}(t)+\int_{-\infty}^{t} F_{2}(t-s) N_{1}(s) d s\right],
\end{array}\right\}
$$

where $r_{1}, r_{2}, \delta_{1}, \delta_{2}$ are positive constants and $F_{1}, F_{2}$ are nonnegative continuous delay kernels defined and integrable on $[0, \infty)$. (5.1) has no predator self-regulating negative feedback. Brelot [6] considered a modified format of (5.1) in the form

$$
\left.\begin{array}{l}
\frac{d N_{1}(t)}{d t}=N_{1}(t)\left[r_{1}-\lambda_{1} N_{1}(t)-\delta_{1} N_{2}(t)-\int_{-\infty}^{t} F_{1}(t-s) N_{2}(s) d s\right], \\
\frac{d N_{2}(t)}{d t}=N_{2}(t)\left[-r_{2}-\lambda_{2} N_{2}(t)+\delta_{2} N_{1}(t)+\int_{-\infty}^{t} F_{2}(t-s) N_{1}(s) d s\right],
\end{array}\right\}
$$

which has terms representing intraspecific competition among both predator and prey species. Under suitable conditions on the various parameters in (5.2) one can show that (5.2) has a (locally) asymptotically stable steady state. A number of integrodifferential equation models of the type (5.1) and (5.2) have been investigated by Cushing [12].

Starting from Hutchinson's (1948) [19] delay logistic equation, May [23] has proposed the following system

$$
\left.\begin{array}{l}
\frac{d N_{1}(t)}{d t}=r N_{1}(t)\left[1-\frac{N_{1}(t-\tau)}{K}\right]-\alpha N_{1}(t) N_{2}(t), \\
\frac{d N_{2}(t)}{d t}=-b N_{2}(t)+\beta N_{2}(t) N_{1}(t),
\end{array}\right\}
$$

where $r, \tau, K, \alpha, \beta, b$ are positive constants; (5.3) contains a single discrete delay; one can modify (5.3) and incorporate a continuously distributed delay in it so that (5.3) can be written as (May [23])

$$
\left.\begin{array}{l}
\frac{d N_{1}(t)}{d t}=r N_{1}(t)\left[1-\int_{-\infty}^{t} F(t-s) N_{1}(s) d s\right]-\alpha N_{1}(t) N_{2}(t), \\
\frac{d N_{2}(t)}{d t}=-b N_{2}(t)+\beta N_{1}(t) N_{2}(t) .
\end{array}\right\}
$$


The first model of a prey-predator type which departs from the Kolmogorovtype formulation is due to Wangersky and Cunningham [33] who considered the model system

$$
\left.\begin{array}{l}
\frac{d N_{1}(t)}{d t}=a_{1} N_{1}(t)-b_{1} N_{1}^{2}(t)-c_{1} N_{1}(t) N_{2}(t), \\
\frac{d N_{2}(t)}{d t}=-a_{2} N_{2}(t)+c_{2} N_{\mathrm{i}}(t-\tau) N_{2}(t-\tau),
\end{array}\right\}
$$

where $a_{1}, a_{2}, b_{1}, c_{1}, c_{2}, \tau$ are positive constants; (5.5) means that a duration of $\tau$ time units elapses when an individual prey is killed and the moment when the corresponding addition is made to the predator population. Recently Cushing [13] has considered a model of the form

$$
\left.\begin{array}{l}
\frac{d N_{1}(t)}{d t}=r N_{1}(t)\left[1-\frac{N_{1}(t)}{K}-\alpha N_{2}(t)\right], \\
\frac{d N_{2}(t)}{d t}=-d_{2}^{*}(t)+b N_{1}(t) \int_{0}^{\infty} \delta(a) N_{2}(t-a) e^{-d^{*} a} d a
\end{array}\right\}
$$

where $r, k, \alpha, d^{*}, b$ are positive constants and $\delta$ is related to the age dependent fecundity of the predator species.

With the models (5.1)-(5.6) in the background let us now consider a time delayed model similar to (4.1) in the following form:

$$
\left.\begin{array}{l}
\frac{d N_{1}(t)}{d t}=f_{1}\left(N_{1}\left(t-\tau_{11}\right)\right)-g_{1}\left(N_{1}(t), N_{2}\left(t-\tau_{12}\right)\right), \\
\frac{d N_{2}(t)}{d t}=f_{2}\left(N_{1}\left(t-\tau_{21}\right), N_{2}\left(t-\tau_{22}\right)\right)-g_{2}\left(N_{2}(t)\right),
\end{array}\right\}
$$

where $\tau_{i j}(i, j=1,2)$ are nonnegative constants with $\tau=\max \tau_{i j}, i, j=1,2$; the birth and death rate functions $f_{1}, f_{2}, g_{1}, g_{2}$ satisfy the same conditions as in (4.1)-(4.6). Along with (5.7) we specify the initial conditions as follows:

$$
\left.\begin{array}{l}
N_{1}(s)=\phi_{1}(s)>0, \\
N_{2}(s)=\phi_{2}(s)>0,
\end{array}\right\} \quad s \in[-\tau, 0] .
$$

We will assume that in addition to (4.2) we have for (5.7) that

$$
\frac{\partial f_{2}}{\partial N_{1}}>0, \quad \frac{\partial f_{2}}{\partial N_{2}}>0, \quad f_{2}\left(N_{1}, N_{2}\right)>0 \quad \text { whenever } N_{1}>0, N_{2}>0 .
$$

It is not difficult to show by the methods previously used for the competition system that whenever $N_{1}(s)>0$ and $N_{2}(s)>0$ for $s \in[-\tau, 0]$, solutions of (5.7) exist for all $t \geqslant 0$ and remain nonnegative for all $t \geqslant 0$. Also $\left(\alpha^{*}, \beta^{*}\right)$ of $(4.5)$ is a steady state of (5.7). 
We will discuss the form and meaning of the delays in (5.7) in the next section. The effects of the various delays in (5.7) on the (local) asymptotic stability of $\left(\alpha^{*}, \beta^{*}\right)$ can be derived from the following theorem whose proof is quite similar to that of Theorem 3.1 and hence the details of the proof are omitted.

THEOREM 5.1. In the time delayed prey-predator system (5.7) let the conditions (4.2)-(4.6), (5.8) and (5.9) hold for the birth and death rates; furthermore suppose the conditions (4.10) hold. Then for all nonnegative delays in (5.7), its nontrivial steady state $\left(\alpha^{*}, \beta^{*}\right)$ is (locally) asymptotically stable.

\section{Estimation of decay rates}

We will first derive a convenient sufficient condition for the asymptotic stability of the trivial solution of a linear system of the form

$$
\left.\begin{array}{l}
\frac{d Y_{1}(t)}{d t}=-m_{11} Y_{1}(t)+b_{11} Y_{1}\left(t-\tau_{11}\right)-m_{12} Y_{2}\left(t-\tau_{12}\right), \\
\frac{d Y_{2}(t)}{d t}=-m_{22} Y_{2}(t)-m_{21} Y_{1}\left(t-\tau_{21}\right)+b_{22} Y_{2}\left(t-\tau_{22}\right),
\end{array}\right\} \quad t>0,
$$

where $m_{i j}, b_{i i}(i, j=1,2)$ are positive constants while $\tau_{i j}(i, j=1,2)$ are nonnegative with $\tau=\max \left(\tau_{i j}\right)(i, j=1,2)$. As is shown in the appendix, the following system dominates (6.1);

$$
\begin{aligned}
& \left.\begin{array}{l}
\frac{d Z_{1}(t)}{d t}=-m_{11} Z_{1}(t)+b_{11} Z_{1}\left(t-\tau_{11}\right)+m_{12} Z_{2}\left(t-\tau_{12}\right), \\
\frac{d Z_{2}(t)}{d t}=-m_{22} Z_{2}(t)+m_{21} Z_{1}\left(t-\tau_{21}\right)+b_{22} Z_{2}\left(t-\tau_{22}\right),
\end{array}\right\} \quad t>0, \\
& \quad Z_{i}(s)=\left|Y_{i}(s)\right|, \quad i=1,2 \quad \text { for } s \leqslant 0 \text { for which } Y_{i}(s) \text { is defined. }
\end{aligned}
$$

Thus the asymptotic stability of the trivial solution of (6.2) implies that of (6.1). It is also shown in the appendix that the solutions of (6.2) are nonnegative for all $t \geqslant 0$. We will use the following vector-matrix notation:

$$
\begin{aligned}
& Z=\left(Z_{1}, Z_{2}\right)^{T}, \quad\|Z\|=\left|Z_{1}(t)\right|+\left|Z_{2}(t)\right|=Z_{1}(t)+Z_{2}(t), \\
& B=\left[\begin{array}{ll}
b_{11} & m_{12} \\
m_{21} & b_{12}
\end{array}\right], \quad\|B\|=\max \left\{\begin{array}{l}
b_{11}+m_{21} \\
b_{21}+m_{12}
\end{array}\right\} \\
& A=\left[\begin{array}{ll}
-m_{11} & 0 \\
0 & -m_{22}
\end{array}\right] .
\end{aligned}
$$


The logarithmic norm or the measure of any square matrix $B$, denoted by $\mu(B)$ is defined as follows:

$$
\mu(B)=\lim _{\theta \rightarrow 0+} \frac{\|I+\theta B\|-1}{\theta}
$$

( $I$ being an identity matrix of the same size as $B$ ). The matrix measure compatible with the norms in (́o.3) is given by

$$
\mu(B)=\max _{J}\left[b_{j j}+\sum_{\substack{i=1 \\ i \neq j}}^{2}\left|b_{i j}\right|\right], \quad B=\left(b_{i j}\right) .
$$

We refer to Coppel [11] or Vidyasager [31] for a number of properties of matrix measure. We have shown in the appendix that the derivative of $H\left(Z_{1}, Z_{2}\right)(t)$ (see A.10) satisfies the relation

$$
\frac{d}{d t} H\left(Z_{1}, Z_{2}\right)(t) \leqslant\left[-m_{11}+b_{11}+m_{21}\right] Z_{1}(t)+\left[-m_{22}+b_{22}+m_{12}\right] Z_{2}(t),
$$

and hence we have

$$
\frac{d}{d t} H\left(Z_{1}, Z_{2}\right)(t) \leqslant\{\mu(A)+\|B\|\}\left\{Z_{1}(t)+Z_{2}(t)\right\} .
$$

If we assume for the matrix norm $\|B\|$ as in (6.3) that

$$
\mu(A)+\|B\|<0 \text { or }-\mu(A)>\|B\|
$$

then from the results of the appendix (Lemmas 5 and 6) it will follow that $Z_{1}(t)+Z_{2}(t) \rightarrow 0$ as $t \rightarrow \infty$ and thus we have the following:

LeMmA 6.1. Assume that the coefficients of the linear system (6.1) satisfy the condition (6.7). Then the trivial solution of (6.1) is asymptotically stable.

When (6.7) holds, an estimate for the decay rate of solutions of (6.1) can be obtained as follows. A necessary and sufficient condition for the asymptotic stability of the trivial solution is that all the roots of the characteristic equation

$$
\operatorname{Det} G\left(\lambda, \tau_{i j}\right)=0
$$

have negative real parts where

$$
\begin{aligned}
G\left(\lambda, \tau_{i j}\right) & =\left[\begin{array}{cc}
\lambda+m_{11} & 0 \\
0 & \lambda+m_{22}
\end{array}\right]-\left[\begin{array}{ll}
b_{11} e^{-\lambda \tau_{11}} & -m_{12} e^{-\lambda \tau_{12}} \\
-m_{21} e^{-\lambda \tau_{21}} & b_{22} e^{-\lambda \tau_{22}}
\end{array}\right] \\
& =\lambda I-A-B\left(\lambda, \tau_{i j}\right) \text { (say), }
\end{aligned}
$$


A positive number $\alpha$ (if it exists) is said to be a decay rate of solutions of (6.1) if and only if every root of (6.8) satisfies the condition

$$
\operatorname{Re}(\lambda)+\alpha<0
$$

When (6.11) holds the solution of (6.1) will be of the order of $e^{-\alpha t}$ and hence the supremum of all such $\alpha>0$ satisfying (6.11) will be a desirable parameter to estimate. However such a supremum of $\alpha$ in (6.11) may not be possible to determine since it has to be done directly from (6.8)-(6.10) (see also Section 3). We will obtain an estimate of $\alpha$ (not necessarily the supremum) in (6.11) by the following procedure.

Replace $\lambda$ by $z-\alpha$ in (6.8) and define

$$
G\left(z-\alpha, \tau_{i j}\right)=M(z)=(z-\alpha) I-A-B\left(z-\alpha, \tau_{i j}\right) .
$$

By Lemma 6.1 a sufficient condition for all roots of $M(z)=0$ to have negative real parts or equivalently to satisfy

$$
\operatorname{Re}(\lambda)<-\alpha
$$

is the following;

$$
-\mu(\alpha I+A)>\left\|B_{1}\left(\alpha, \tau_{i j}\right)\right\|,
$$

where

$$
B_{1}\left(\alpha, \tau_{i j}\right)=\left[\begin{array}{ll}
b_{11} e^{\alpha \tau_{11}} & -m_{12} e^{\alpha \tau_{12}} \\
-m_{21} e^{\alpha \tau_{21}} & b_{22} e^{\alpha \tau_{22}}
\end{array}\right] .
$$

Since $\mu(\alpha I+A)=\alpha+\mu(A)$ we have from (6.13) that

$$
\alpha+\mu(A)+\left\|B_{1}\left(\alpha, \tau_{i j}\right)\right\|<0
$$

is a sufficient condition for $\operatorname{Re}(\lambda)<-\alpha$. If we denote the left side of (6.15) by $f\left(\alpha, \tau_{i j}\right)$ we note that

$$
f\left(0, \tau_{i j}\right)=\|B\|+\mu(A)<0 \text { if (6.7) holds; }
$$

also $\partial f / \partial \alpha>0, f$ is continuous and monotonic in $\alpha ; f(\alpha) \rightarrow \infty$ as $\alpha \rightarrow \infty$; thus by the continuity of $f$ on $\alpha \geqslant 0$, there exists an interval of the form $\left[0, \alpha^{*}\right)$ such that

$$
f\left(\alpha, \tau_{i j}\right)<0 \text { for } \alpha \in\left[0, \alpha^{*}\right)
$$

and hence

$$
f\left(\alpha^{*}, \tau_{\imath \jmath}\right)=0 .
$$

thus an upper bound $\alpha^{*}$ of $\alpha$ for which we have $f\left(\alpha, \tau_{i j}\right)<0, \alpha \in\left[0, \alpha^{*}\right)$ can be obtained as the unique (by the monotonicity of $f$ on $\alpha$ ) positive root of

$$
\max \left\{\begin{array}{l}
b_{11} e^{\alpha \tau_{11}}+m_{21} e^{\alpha \tau_{21}} \\
b_{22} e^{\alpha \tau_{22}}+m_{12} e^{\alpha \tau_{12}}
\end{array}\right\}+\alpha-\min \left\{\begin{array}{l}
m_{11} \\
m_{22}
\end{array}\right\}=0
$$


where the maximum in (6.18) is taken over $b_{11}, b_{22}, m_{12}, m_{21}$ and $\tau_{i j}(i, j=1,2)$. A numerical procedure can be devised to solve equations of the form (6.18) in concrete cases. If one is interested in a smaller bound on the decay rate it can be obtained as the unique root of

$$
\|B\| e^{\alpha \tau}+\alpha+\mu(A)=0, \quad \tau=\max _{i, j} \tau_{t,} .
$$

It will follow from (6.19) than an increase in $\tau$ will reduce the value of the root of (6.19). We remark that since our estimate $\alpha^{*}$ of decay rate is based on a sufficient condition of the form (6.7), we can conclude that the solutions of (6.1) will converge at least as fast as $e^{-\alpha^{*} t}$ where $\alpha^{*}$ is the unique root of either (6.18) or (6.19).

\section{Ecological and biological implications}

We have formulated a class of two species population model systems starting from the elementary principle that the rate of change of a population equals the birth rate minus the death rate. This is a departure from the conventional model building in population ecology where one usually starts from the Malthusian formulation, now known as the Kolmogorov formulation in the nonlinear context. In our analysis of the models proposed we have obtained sufficient conditions for the global existence of nonnegative solutions of the model systems (this is trivial in the Kolmogorov formulation) and the other desirable properties such as the existence of suitable steady states. The two classes of models proposed for competition and predation contain intraspecific self-regulating negative feedback terms. While there is general consensus among population biologists that each species is under the action of a self-regulating negative feedback force, opinions and arguments differ regarding the mechanism by which such an effect is realised; we refer for more details on this aspect to a collection of articles compiled by Tamarin [30].

Our main result is the following: if a self-regulating negative feedback mechanism (intraspecific competition) can act without time delay and if such an effect can keep an ecosystem (a community of one or more species of populations) asymptotically stable (at least locally) about a nontrivial steady state, then time delays of any magnitude in any other interaction involving positive or negative feedbacks cannot destabilise such a stable ecosystem (in a mathematical sense); that is such delays cannot cause a Hopf-type bifurcation leading to persistent and undamped fluctuations. The delay-induced bifurcations to oscillations in population models have been investigated by many authors. 
Qualitatively our result is not new to the literature; the effects of delays on otherwise stable and unstable systems have been considered by Cushing [12] where (pages 35-39) he has shown that an otherwise unstable system can be made less unstable by delays. Such an effect of delays on unstable systems appear to have been first proposed by Beddinton and May [2] who have also shown that delays can make stable systems less stable leading to a conclusion "time delays are not necessarily destabilising". It is also shown by Cushing ([12], pages 75-103) that while time delays cannot stabilise (in the mathematical sense) an otherwise unstable two species system in competition or mutualism, time delays in three species systems can stabilise otherwise unstable systems (even in a mathematical sense). Cushing and Saleem [14] have formulated a model where delays in the positive feedback can have a stabilising effect; Hale [18] discusses briefly the stabilising nature of delays and gives an example to show that an increase in delays can make an unstable system stable. Thus for a better understanding of the effects of delays on otherwise stable systems one has to estimate the rate of decay of perturbations (or the characteristic return time) in terms of the parameters of the system especially when the delays do not cause a mathematical instability. This aspect has been investigated by Brauer $[4,5]$ for models with a single delay using mainly the characteristic equation. By a procedure slightly different from that of the characteristic equation we have proposed (Section 6) a method for the estimation of decay rate of perturbations when there are multiple delays. Since many species systems with multiple delays cannot be analysed (due to complexity) by the characteristic equation, our methods using comparison lead to easily verifiable sufficient conditions. The sufficient conditions obtained are in the form of a diagonal dominance type; these conditions can be weakened to quasi-diagonal dominance type as in Ladde [20], Siljak [29], Anderson [1], Lewis and Anderson [21,22] (Ladde's result [20] is delay dependent due to the fact that delay appears in self-regulation also). Verification of quasi-diagonal dominance conditions in arbitrary matrices (except for Metzler matrices) is an impossible task and ecologically quasi-diagonal dominance is hard to interpret.

It follows from our discussion that those population systems which display delay induced instability leading to permanent and undamped oscillations should have one or more of the following aspects:

(1) time delays are in intraspecific interaction terms (and possibly in other terms also);

(2) intraspecific self-regulation terms are absent in density dependent form in one or more populations of the system;

(3) intraspecific negative feedback effects are not strong enough to maintain the stability of the relevant steady state of the nondelayed system.

We can now infer how the delay induced stability leads to fluctuations; a primary mechanism of such fluctuations can be described at least theoretically as 
follows: in its attraction of a system to a steady state, intraspecific self-regulation fails to be effective and the system overshoots the steady state and at such higher densities intraspecific competition becomes operationally effective leading the system to undershoot the steady state and this process is repeated again and again resulting in undamped oscillations about the steady state. It follows from our results that if intraspecific competition is sufficiently strong, the system cannot become oscillatory even when there are time delays, so long as they do not interfere with the strength of the intraspecific self-regulation.

We are thus led to the question of existence and importance of intraspecific competition in population systems. In the introduction to an anthology of articles by several authors, Tamarin [30] begins with the statement "all animal populations have the reproductive capacity to increase without limit, yet none do" and in his concluding remarks writes "this leaves us with only one great concept in the area of population regulation: that all animal populations are regulated".

In his discussion on the effect of competition on the densities of animal populations, Nicholson ([25], page 147) has reached the conclusion "Intraspecific competition automatically regulates the severity of its action to the requirements of each population, provided the inherent resistance of the environment is sufficiently low to permit the species to exist." In order to look for a mechanism by which intraspecific competition plays its role of decreasing growth rate as population density increases, Calhoun [7] set up an experiment with Norway rats (Rattus norvegicus) and found that increased density leads to increased hostility which disturbs the psychology and physiology causing decreased reproductive output. Many other mechanisms by which intraspecific competition self-regulates a population have been put forward by a number of authors (see Tamarin [30]).

If we accept the view that intraspecific competition is really predominant in population regulation, then we can understand how and why time delays destabilise stable systems leading to oscillations; time delays render intraspecific competitive regulation temporarily weak so that self-regulatory forces become temporarily insufficient. If we now agree that all population models should incorporate self-regulating intraspecific competition terms in sufficient strength along with other trophic interactions then it is not fair to say "time delays destabilise" and we note from our analysis that it is usually a failure in self-regulation which can cause instability rather than delays by themselves.

We will give one example to illustrate our point. Consider a prey-predator system in which the prey is self-regulating by a logistic load as follows:

$$
\begin{aligned}
& \frac{d x}{d t}=r\left(x-\frac{x^{2}}{K}\right)-\alpha x y=b_{1}(x)-m_{1}(x, y) \quad \text { (say), } \\
& \frac{d y}{d t}=\beta x y-\delta y=b_{2}(x, y)-m_{2}(y) \quad \text { (say), }
\end{aligned}
$$


where $K, \alpha, \beta, \delta$ are positive constants such that the steady state $\left(x^{*}, y^{*}\right)$ exists where

$$
x^{*}=\frac{\delta}{\beta} ; \quad y^{*}=\frac{r}{\alpha}\left(1-\frac{\delta}{k \beta}\right)>0 .
$$

In this example the predator self-regulation $\left(\partial m_{2} / \partial y\right)$ is not sufficiently strong in the sense

$$
\left(\frac{\partial m_{2}}{\partial y}\right)_{\left(x^{*}, y^{*}\right)} \ngtr\left(\frac{\partial b_{2}}{\partial y}+\frac{\partial m_{1}}{\partial y}\right)_{\left(x^{*}, y^{*}\right)} .
$$

If in such a model there is a time delay in the predator birth rate, such a delay can lead to instability of $\left(x^{*}, y^{*}\right)$ setting up undamped oscillations. If however we modify the predator growth rate by the inclusion of a density dependent self-regulation so that

$$
\frac{d y}{d t}=\beta x y-\delta y-\eta y^{2}
$$

for some positive constant $\eta$ then it is possible, depending on $\eta$, for such a modified system to remain stable without oscillations even if there is a time delay in the predator birth rate. We have another completely different justification to have $\eta y^{2}$ in the predator dynamics; for instance if the prey is removed from the food chain, we expect the predator density $y$ to drop to zero in finite time; but without a term of the type $\eta y^{2}$ this cannot happen. While this example serves to illustrate an aspect of our result, our advocacy of the significance is not based on this example.

We have derived our conclusion that "time delays need not destabilise" in models with several discrete delays. However we have provided in the appendix the necessary tools (Lemmas $3,4,5$ ) to prove all our results when the relevant delays are all (or some) continuously distributed. Some authors (Caswell [9], May [23], Cushing [12]) appear to regard continuously distributed delays as ecologically more realistic although the only evidence for this is the work reported by Caperon [8] who tried a discrete and continuous delay model to fit the data obtained when he subjected algae to a variable nitrate environment and obtained a better fit for his data with a continuous delay. The question of whether a discrete or continuous delay is more realistic may well depend on what biological process is being modelled. For instance an increased supply of some resource at some point in time may trigger reproduction at a particular moment in time later irrespective of the conditions of the resource during the intervening period. If the duration of increased resource supply necessary to elicit such a response is short compared to the intervening period before the reproduction occurs, then a discrete time delay may be appropriate. In a large number of cases of animal 
populations, the past dietary and nutritional history of an animal over a long period plays an influential role in determining the current behaviour of the animal and in such cases continuous time delays will be appropriate.

In conclusion the following comments are appropriate. The time delays in the birth rates of competition models correspond to maturation delays since the current birth rate is usually due to a contribution of those mature animals living a few units of time earlier depending on the type of animal or organism; the time delays appearing in interspecific interactions can be due to the fact that in order to successfully interact and compete interferentially a developmental maturity may be necessary. In the case of predation, delays in the birth rate of prey can be due to delayed maturation while that in the birth rate of a predator is due to gestation delay with delayed fecundity; the delays in the predation rate are again as before due to the maturity needed for a predator to successfully organise and succeed in an attack.

In our analysis we have assumed throughout a temporally uniform environment neglecting dispersal of populations and their age structure. We expect our results to hold under suitable conditions both in temporally periodic environments with the steady state of a constant environment being replaced by a periodic solution (nonstationary steady state) and when the effects of dispersion and age structures are considered. It is worthwhile to modify our purely ecological models so as to provide them with a genetic foundation and examine the effects of time delays since forces of selection act with some time delay. Such a generalisation will definitely lead to a better understanding of "how populations regulate themselves".

\section{Appendix}

First we derive a comparison result.

LEMMA 1. Consider the delay differential system

$$
\left.\begin{array}{l}
\frac{d Y_{1}(t)}{d t}=-m_{11} Y_{1}(t)+b_{11} Y_{1}\left(t-\tau_{11}\right)-m_{12} Y_{2}\left(t-\tau_{12}\right), \\
\frac{d Y_{2}(t)}{d t}=-m_{22} Y_{2}(t)-m_{21} Y_{1}\left(t-\tau_{21}\right)+b_{22} Y_{2}\left(t-\tau_{22}\right),
\end{array}\right\}
$$

where the $m_{i j}, b_{i j}(i, j=1,2)$ are positive while $\tau_{i j}(i, j=1,2)$ are nonnegative with $\tau=\max \tau_{i j}, i, j=1,2$. Then

$$
\left.\begin{array}{l}
\frac{d\left|Y_{1}(t)\right|}{d t} \leqslant-m_{11}\left|Y_{1}(t)\right|+b_{11}\left|Y_{1}\left(t-\tau_{11}\right)\right|+m_{12}\left|Y_{2}\left(t-\tau_{12}\right)\right|, \\
\frac{d\left|Y_{1}(t)\right|}{d t} \leqslant-m_{22}\left|Y_{2}(t)\right|+m_{21}\left|Y_{1}\left(t-\tau_{21}\right)\right|+b_{22}\left|Y_{2}\left(t-\tau_{21}\right)\right|,
\end{array}\right\}
$$


where

$$
\frac{d}{d t}\left|Y_{i}(t)\right|=\left\{\begin{array}{cc}
\frac{d Y_{i}(t)}{d t} & \text { if } Y_{i}(t) \geqslant 0 \\
-\frac{d Y_{i}(t)}{d t} & \text { if } Y_{i}(t)<0
\end{array}\right\} \quad i=1,2
$$

The proof is by direct verification.

LEMMA 2. Consider now (A.2) and the following system

$$
\left.\begin{array}{l}
\frac{d Z_{1}(t)}{d t}=-m_{11} Z_{1}(t)+b_{11} Z_{1}\left(t-\tau_{11}\right)+m_{12} Z_{2}\left(t-\tau_{12}\right), \\
\frac{d Z_{2}(t)}{d t}=-m_{22} Z_{2}(t)+m_{21} Z_{1}\left(t-\tau_{21}\right)+b_{22} Z_{2}\left(t-\tau_{22}\right),
\end{array}\right\}
$$

together with the initial conditions

$$
Z_{\imath}(s)>\left|Y_{\imath}(s)\right|, \quad s \in[-\tau, 0], \quad i=1,2 .
$$

Then

$$
\left|Y_{t}(t)\right|<Z_{i}(t) \text { for } t \geqslant 0 \text { and } i=1,2 .
$$

Proof. Let $U_{i}(t)=Z_{i}(t)-\left|Y_{i}(t)\right|, i=1,2, t \geqslant-\tau$. Then we have from (A.2) and (A.4),

$$
\left.\begin{array}{l}
\frac{d U_{1}(t)}{d t} \geqslant-m_{11} U_{1}(t)+b_{11} U_{1}\left(t-\tau_{11}\right)+m_{12} U_{2}\left(t-\tau_{12}\right), \\
\frac{d U_{2}(t)}{d t} \geqslant-m_{22} U_{2}(t)+m_{21} U_{1}\left(t-\tau_{21}\right)+b_{22} U_{2}\left(t-\tau_{22}\right),
\end{array}\right\} \quad t>0,
$$

with

$$
U_{i}(s)=Z_{i}(s)-\left|Y_{i}(s)\right|>0, \quad i=1,2, s \in[-\tau, 0] .
$$

Consider (A.7)-(A.8) first on the interval [0, $\tau]$ : It will follow from (A.7) that $U_{1}(t) \geqslant U_{1}(0) \exp \left[-m_{11} t\right]$

$$
+\int_{0}^{t}\left[b_{11} U_{1}\left(s-\tau_{11}\right)+m_{12} U_{2}\left(s-\tau_{12}\right)\right] \exp \left[-m_{11}(t-s)\right] d s>0
$$

for all $t \in\left[0, \tau^{*}\right]$ since $U_{i}(s)>0$ on $\left[-\tau^{*}, 0\right]$ where $\tau^{*}=$ positive minimum of $\left(\tau_{11}, \tau_{12}, \tau_{21}, \tau_{22}\right)$. Similarly $U_{2}(t)>0$ for $t \in\left[0, \tau^{*}\right]$. Now by a similar argument it will follow that $U_{1}(t)>0, U_{2}(t)>0$ for $t \in\left[\tau^{*}, 2 \tau^{*}\right]$. Repeating the above procedure we obtain the result (A.6).

It follows from Lemmas 1 and 2 that in order to prove Theorem 3.1 it is enough to prove that $Z_{i}(t) \rightarrow 0$ as $t \rightarrow \infty$ for $i=1,2$ which we will do as follows. 
Proof of Theorem 3.1. Define a Lyapunov-like function

$$
\begin{aligned}
H\left(Z_{1}, Z_{2}\right)(t)= & Z_{1}(t)+Z_{2}(t) \\
& +\int_{t-\tau_{11}}^{t} b_{11} Z_{1}(s) d s+\int_{t-\tau_{12}}^{t} m_{12} Z_{2}(s) d s \\
& +\int_{t-\tau_{21}}^{t} m_{21} Z_{1}(s) d s+\int_{t-\tau_{22}}^{t} b_{22} Z_{22}(s) d s .
\end{aligned}
$$

Since each term of $H$ in (A.10) is nonnegative for $t>0$, if we show that $H \rightarrow 0$ as $t \rightarrow \infty$ it will follow from Lemma 2 that

$$
0 \leqslant\left|Y_{l}(t)\right| \leqslant Z_{l}(t) \rightarrow 0 \quad \text { as } t \rightarrow \infty
$$

implying that the perturbations $Y_{1}, Y_{2}$ governed by (A.1) approach zero as $t \rightarrow \infty$, and this will prove our result. Calculate the rate of change of $H$ along the solutions of (A.4) so that

$$
\begin{aligned}
\frac{d H}{d t} & =\frac{\partial H}{\partial t}+\frac{\partial H}{\partial Z_{1}} \frac{d Z_{1}}{d t}+\frac{\partial H}{\partial Z_{2}} \frac{d Z_{2}}{d t} \\
& =-\left[Z_{1}(t)\left\{m_{11}-\left(b_{11}+m_{21}\right)\right\}+Z_{2}(t)\left\{m_{22}-\left(b_{22}+m_{12}\right)\right\}\right]<0
\end{aligned}
$$

on using (2.10) since $Z_{1}(t), Z_{2}(t)$ are positive for all $t>0$. Thus $H\left(Z_{1}, Z_{2}\right)(t) \rightarrow 0$ as $t \rightarrow \infty$ since $H$ is bounded below by zero; it follows from Lemma 6 below that $Z_{1}(t) \rightarrow 0, Z_{2}(t) \rightarrow 0$ as $t \rightarrow \infty$. The proof is complete.

The following set of results are intended to be a guide to prove results similar to the above in the case of continuously distributed delays. An analogue of Lemma 1 is the following.

Lemma 3. Let $k_{i j}(s), s \in[0, \infty), i, j=1,2$, denote nonnegative continuous functions integrable on $[0, \infty)$ normalised such that

$$
\int_{0}^{\infty} s k_{i j}(s) d s<\infty ; \quad \int_{0}^{\infty} k_{i j}(s) d s=1, \quad i, j=1,2 .
$$

Consider the integrodifferential system

$$
\begin{aligned}
\frac{d Y_{1}(t)}{d t}= & -m_{11} Y_{1}(t)+b_{11} \int_{0}^{\infty} k_{11}(s) Y_{1}(t-s) d s \\
& -m_{12} \int_{0}^{\infty} k_{12}(s) Y_{2}(t-s) d s, \\
\frac{d Y_{2}(t)}{d t}= & -m_{22} Y_{2}(t)-m_{21} \int_{0}^{\infty} k_{21}(s) Y_{1}(t-s) d s \\
& +b_{22} \int_{0}^{\infty} k_{22}(s) Y_{2}(t-s) d s,
\end{aligned}
$$


where $m_{i j}, b_{i j}(i, j=1,2)$ are positive constants. Then

$$
\left.\begin{array}{rl}
\frac{d\left|Y_{1}(t)\right|}{d t} \leqslant & -m_{11}\left|Y_{1}(t)\right|+b_{11} \int_{0}^{\infty} k_{11}(s)\left|Y_{1}(t-s)\right| d s \\
& +m_{12} \int_{0}^{\infty} k_{12}(s)\left|Y_{2}(t-s)\right| d s \\
\frac{d\left|Y_{2}(t)\right|}{d t} \leqslant & -m_{22}\left|Y_{2}(t)\right|+m_{21} \int_{0}^{\infty} k_{21}(s)\left|Y_{1}(t-s)\right| d s \\
& +b_{22} \int_{0}^{\infty} k_{22}(s)\left|Y_{2}(t-s)\right| d s
\end{array}\right\}
$$

where $d\left|Y_{i}(t)\right|$ dt is defined by (A.3).

Proof is again by direct verification.

The proof of the following lemma is similar to that of Lemma 2 above.

LEMMA 4. Consider (A.14) and the following integrodifferential system:

$$
\left.\begin{array}{rl}
\frac{d Z_{1}(t)}{d t}= & -m_{11} Z_{1}(t)+b_{11} \int_{0}^{\infty} k_{11}(s) Z_{1}(t-s) d s \\
& +m_{12} \int_{0}^{\infty} k_{12}(s) Z_{2}(t-s) d s \\
\frac{d Z_{2}(t)}{d t}= & -m_{22} Z_{2}(t)+m_{21} \int_{0}^{\infty} k_{21}(s) Z_{1}(t-s) d s \\
& +b_{22} \int_{0}^{\infty} k_{22}(s) Z_{2}(t-s) d s,
\end{array}\right\}
$$

together with the initial conditions

$$
\infty>Z_{i}(s)>\left|Y_{i}(s)\right| \text { for } s \in(-\infty, 0] \text {. }
$$

Then $\left|Y_{i}(t)\right|<Z_{i}(t)$ for $t \geqslant 0, i=1,2$.

The following lemma will be useful to prove a result analogous to that of Theorem 3.1 corresponding to a class of continuously distributed time delays.

LEMmA 5. Let $Z_{1}(t), Z_{2}(t)$ be the solutions of (A.15)-(A.16) where $\left(Y_{1}, Y_{2}\right)$ corresponds to an arbitrary but fixed solution of (A.13). Then as a consequence of Lemma 4 it will follow that

$$
0 \leqslant\left|Y_{i}(t)\right| \leqslant Z_{i}(t) \rightarrow \infty \text { as } t \rightarrow \infty, \quad i=1,2,
$$


whenever

$$
\left\{\begin{array}{l}
m_{11}>b_{11}+m_{21}, \\
m_{22}>b_{22}+m_{12}
\end{array}\right\}
$$

Proof. Define a Lyapunov-like function $H$ where

$$
\begin{aligned}
H\left(Z_{1}, Z_{2}\right)(t)= & Z_{1}(t)+Z_{2}(t)+b_{11} \int_{0}^{\infty} k_{11}(u)\left(\int_{t-u}^{t} Z_{1}(s) d s\right) d u \\
& +m_{12} \int_{0}^{\infty} k_{12}(u)\left(\int_{t-u}^{t} Z_{2}(s) d s\right) d u \\
& +m_{21} \int_{0}^{\infty} k_{21}(u)\left(\int_{t-u}^{t} Z_{1}(s) d s\right) d u \\
& +b_{22} \int_{0}^{\infty} k_{22}(u)\left(\int_{t-u}^{t} Z_{2}(s) d s\right) d u
\end{aligned}
$$

where $\left(Z_{1}, Z_{2}\right)$ is any solution of (A.15)-(A.16) for arbitrary $\left(Y_{1}, Y_{2}\right)$ defined on $(-\infty, 0]$. Computing the rate of change of $H$ in (A.19) along the solutions of (A.15) we derive that (on using (A.12))

$$
\begin{aligned}
\frac{d H\left(Z_{1}, Z_{2}\right)(t)}{d t} & =-\left[Z_{1}(t)\left\{m_{11}-\left(b_{11}+m_{21}\right)\right\}+Z_{2}(t)\left\{m_{22}-\left(b_{22}+m_{12}\right)\right\}\right] \\
& <0 \quad \text { (using (A.18)) for } Z_{1}(t)>0 \text { and } Z_{2}(t)>0 .
\end{aligned}
$$

By Lemma 6 below the result will follow.

LemMa 6. Let $H(t)=H\left(Z_{1}, Z_{2}\right)(t)$ be as defined in (A.10). Suppose

$$
m_{11}>b_{11}+m_{21} \text { and } m_{22}>b_{22}+m_{12} \text {. }
$$

Then

$$
Z_{1}(t)+Z_{2}(t) \rightarrow 0 \text { as } t \rightarrow \infty .
$$

Proof. Suppose (A.21) does not hold; then there exists at least one sequence $\left\{t_{n}\right\} \rightarrow \infty$ as $n \rightarrow \infty$ such that

$$
\left[m_{11}-\left(b_{11}+m_{21}\right)\right] Z_{1}\left(t_{n}\right)+\left[m_{22}-\left(b_{22}+m_{12}\right)\right] Z_{2}\left(t_{n}\right)>\varepsilon
$$

for some sufficiently small positive number $\varepsilon$ and hence

$$
\frac{d H\left(t_{n}\right)}{d t}<-\varepsilon, \quad n=1,2,3, \ldots .
$$

By the continuity and differentiability of $H$ with respect to $t$,

$$
H\left(t_{n}-\varepsilon\right)-H\left(t_{n}\right)=\frac{d H\left(t^{*}\right)}{d t}(-\varepsilon), \quad t_{n}-\varepsilon<t^{*}<t_{n} .
$$


If $\varepsilon$ is small we will have

$$
-\frac{d H\left(t^{*}\right)}{d t}>\varepsilon / 2
$$

so that

$$
\begin{aligned}
H\left(t_{n}\right) \leqslant & H\left(t_{n}-\varepsilon\right)-\varepsilon^{2} / 2 \\
\leqslant & H\left(t_{n-1}\right)-\varepsilon^{2} / 2 \\
\leqslant & H\left(t_{n-2}\right)-2\left(\varepsilon^{2} / 2\right) \\
\leqslant & H\left(t_{n-2}\right)-2\left(\varepsilon^{2} / 2\right) \\
& \vdots \\
\leqslant & H\left(t_{0}\right)-n\left(\varepsilon^{2} / 2\right) ; \quad t_{0}=0 .
\end{aligned}
$$

(A.12) and Lemma 5 will imply that $H\left(t_{0}\right)<\infty$ for any $t_{0}>0$. If $n$ is sufficiently large, it will follow that $H\left(t_{n}\right)<0$ since $H\left(t_{0}\right)<\infty$, which contradicts the nonnegativity of $H$ for all $t \geqslant 0$. Hence $Z_{1}(t)+Z_{2}(t) \rightarrow 0$ as $t \rightarrow \infty$ implying the asymptotic stability of the trivial solution of (A.1) (since $Z_{l}$ dominates $Y_{l}$ ).

We note the results of Lemma 5 and 6 will remain valid if (A.18) is replaced by the condition $\mu(A)+\|B\|<0$ where $A$ and $B$ are defined as in (6.3).

\section{References}

[1] B. D. O. Anderson, "Time delays in large scale systems", Proc. 18th Conf. on Decision and Control, (1979).

[2] J. R. Beddington and R. M. May, "Time delays are not necessarily destabilising”, Math. Brosci. 27 (1975), 109-117.

[3] R. Bellman and K. L. Cooke, Differential difference equations (Academic Press, New York, 1963).

[4] F. Brauer, "Stability of population models with delay", Math. Biosci. 33 (1977), 345-358.

[5] F. Brauer, "Decay rates for solutions of a class of differential difference equations", SIAM J. Math. Anal. 10 (1979), 783-788.

[6] M. Brelot, "Sur le problème biologique héréditaire de deux especès devorante et dévore", Ann. Mat. Pura Appl. 9 (1931), 58-74.

[7] J. B. Calhaun, "A method for self control of population growth among mammals living in the wild", Science 109 (1949), 333-335.

[8] J. Caperon, "Time lag in population growth response of Isochrysis galbana to a variable nitrate environment", Ecology 50 (1969), 188-192.

[9] H. Caswell, "A simulation study of a time lag population model”, J. Theoret. Biol. 34 (1972), 419-439.

[10] D. S. Cohen, E. Coutsias and J. C. Neu, "Stable oscillations in single species growth models with hereditary effects", Math. Biosci. 44 (1979), 255-268.

[11] W. A. Coppel, Stability and asymptotic behaviour of differential equations (D. C. Heath and Company, Boston, Mass., 1965). 
[12] J. M. Cushing, "Integrodifferential equations with delay models in population dynamics", Lecture Notes in Biomathematics 20 (Springer-Verlag, Berlin, 1977).

[13] J. M. Cushing, "Volterra integrodifferential equations in population dynamics", Proc. Centro Internazionale Matematico Estivo Summer Session on Mathematics in Biology (1979).

[14] J. M. Cushing and M. Saleem, "A predator prey model with age structure", J. Math. Biol. 14 (1982), 231-250.

[15] K. Gopalsamy and B. D. Aggarwala, "Limit cycles in two species competition models with time delays", J. Austral. Math. Soc. Ser. B 22 (1980), 148-160.

[16] K. Gopalsamy. "A delay induced bifurcation to oscillations", J. Math. Phys. Sct. 16 (1982), 469-488.

[17] J. K. Hale, Theory of functional differential equatıons (Springer-Verlag, New York, 1977).

[18] J. K. Hale, "Nonlinear oscillations in equations with delays", in Lectures in Appled Math. 17 (Amer. Math. Soc., Providence, R.I., 1979), 157-185.

[19] G. E. Hutchinson, "Circular causal systems in ecology", Ann. N. Y. Acad. Scl. 50 (1948), $221-246$.

[20] G. S. Ladde, "Stability of model ecosystems with time delay", J. Theoret. Biol. 61 (1976), 1-13.

[21] R. M. Lewis and B. D. O. Anderson, "Necessary and sufficient conditions for delay independent stability of linear autonomous systems", IEEE Trans. Automat. Control 25 (1980), 735-739.

[22] R. M. Lewis and B. D. O. Anderson, "Insensitivity of a class of nonlinear compartmental systems to the introduction of arbitrary time delays", IEEE Trans. Circults and Systems 27 (1980), 604-612.

[23] R. M. May, "Time delay versus stability in population models with two and three trophic levels", Ecology 54 (1973), 315-325.

[24] R. M. May, Stability and complexity in model ecosystems (Princeton Unjversity Press, Princeton, N.J., 1974).

[25] A. J. Nicholson, "The balance of animal populations", J. Anim. Ecol. (1933), 132-178.

[26] A. Rescigno and I. W. Richardson, "The struggle for life. I: Two species", Bull. Math. Biophys. 29 (1967), 377-388.

[27] R. E. Ricklefs, Ecology (Chiron Press, Newton, Mass., 1974).

[28] A. Shibata and N. Saito, "Time delays and chaos in two competing species", Math. Biosci. 51 (1980), 199-211.

[29] D. D. Siljak, "When is a complex ecosystem stable?", Math. Biosci. 25 (1975), 25-50.

[30] R. H. Tamarin (ed.), Population regulation (Dowden, Hutchinson and Ross., Inc., Stroudsburg, Pa., 1978).

[31] M. Vidyasager, Nonlınear system analysis (Prentice-Hall, Englewood Cliffs, N. J., 1978).

[32] V. Volterra, Leçons sur la théorie mathématıque de la lutte pour la vie (Gauthier-Villars, Paris, 1931).

[33] P. J. Wangersky and W. J. Cunningham, “Time lag in population models", Cold Spring Harbor Symp. Qual. Biol. 22 (1957), 329-338. 